\title{
Soft Sensing and Optimal Power Control for Cognitive Radio
}

\author{
Sudhir Srinivasa and Syed Ali Jafar \\ Electrical Engineering and Computer Science \\ University of California Irvine, Irvine, CA 92697-2625 \\ Email:sudhirs@uci.edu,syed@uci.edu
}

\begin{abstract}
We consider a cognitive radio system where the secondary transmitter varies its transmit power based on all the information available from the spectrum sensor. The operation of the secondary user is governed by its peak transmit power constraint and an average interference constraint at the primary receiver. Without restricting the sensing scheme (total received energy, or correlation etc), we characterize the power adaptation strategies that maximize the secondary user's SNR and capacity. We show that, in general, the capacity optimal power adaptation requires decreasing the secondary transmit power from the peak power to zero in a continuous fashion as the probability of the primary user being present increases. We find that power control that maximizes the SNR is binary, i.e., if there is any transmission, it takes place only at the peak power level. Numerical results for common spectrum sensing schemes show that the SNR and capacity maximizing schemes can be significantly different.
\end{abstract}

\section{INTRODUCTION}

In recent years, increased dependence on diverse wireless technologies has generated a huge demand for more bandwidth. While the traditional 'divide and set aside' approach to spectrum regulation ensures that the licensed (primary) users cause minimal interference to each other, it has created a crowded spectrum with most frequency bands already assigned to different licensees [1]. The term 'cognitive radio' encompasses several techniques [2]-[7] that seek to overcome the spectral shortage problem by enabling secondary (unlicensed) wireless devices to communicate without interfering with the primary users. Our work will exclusively focus on the 'interweave' (interference avoidance) approach [4]-[7] to cognitive radio, wherein the secondary radio periodically monitors and intelligently detects occupancy in the different frequency bands and then opportunistically communicates over the spectrum holes with minimal interference to the active primary users.

The main challenge to opportunistic communication lies in striking a balance between the conflicting goals of minimizing the interference to the primary users and maximizing the performance of the secondary users [8], [9]. This issue can be addressed by adapting the transmit power based on the reliability of the sensed information. Prevalent cognitive radio models implement the primary user sensing as a binary hypothesis test. The spectrum sensor outputs a binary decision ( 0 or 1$)$ that indicates whether or not the PU has been detected. The secondary transmit power depends on the sensed signals only through this binary decision. This kind of power adaptation is based on hard decisions. In the absence of channel knowledge at the transmitter, it involves transmitting at two power levels - zero when the primary user is detected and at the peak power when no primary radio is deemed present - thereby simplifying implementation at the secondary transmitter. With binary detection and binary power control, minimizing interference reduces to satisfying a missed detection probability constraint while maximizing the secondary performance reduces to satisfying a false alarm probability constraint.

We emphasize that there is a loss of information in translating the (analog) sensed signals to a binary decision. The motivation behind our work stems from the possibility that this information (soft information) can be used through sophisticated (continuous) power control to improve the system performance. For example, instead of the simple two level power switching (zero or peak power), one can have a power adaptation scheme where the transmit power increases continuously from 0 to the peak power $P_{\max }$ as a function of the sensed information. With soft sensing based continuous power adaptation, the notions of missed detection and false alarm probabilities are irrelevant. This generalized setting brings us back to the ultimate goals of minimizing the interference to the primary users and maximizing the performance (SNR or capacity) of the secondary users. While binary detection and power control are interesting for their simplicity, we explore soft sensing and continuous power adaptation in order to identify optimal cognitive radio design principles.

We consider a cognitive radio system where the secondary transmitter varies its transmit power based on the value of the sensing metric. We assume a peak power constraint at the secondary transmitter and an average interference constraint at the primary receiver. The following is a summary of our main results:

- For an arbitrary sensing scheme, we identify and characterize the power adaptation strategies that maximize the SNR at the secondary receiver and the capacity of the secondary user.

- Binary (hard) power adaptation is optimal for SNR regardless of the type of sensing metric, i.e., the SNR optimal power adaptation policy mandates that transmissions take place only at the peak power.

- In general, we find that the capacity optimal power adaptation is not binary and involves transmissions at non-boundary power levels between zero and the peak power.

- With analytical and numerical results, we show that the SNR optimal and capacity optimal power adaptation schemes are similar for common spectrum sensing schemes. 


\section{SySTEM MODEL}

Consider a communication system with a primary transmitter (PR) and primary receiver (PR) licensed to operate over a certain frequency band as shown in Figure 1. The primary user (primary transmitter - receiver pair, PU) activity follows a block static model with a coherence time $T_{c}$ and an $O N$ probability of $\alpha$, i.e., the primary user remains active (ON state) or inactive (OFF state) for a period of $T_{c}$ channel uses after which it switches to an independent ON (or OFF) state (with a probability $\alpha$ of switching to the $\mathrm{ON}$ state). We assume that the primary transmitter uses a Gaussian codebook with an average power $P_{t}$ for the primary transmissions.

To allow for higher spectral efficiencies, the channel is also open to be used by a cognitive user (secondary transmitter (ST) - secondary receiver (SR) pair, SU) as Figure 1 shows.

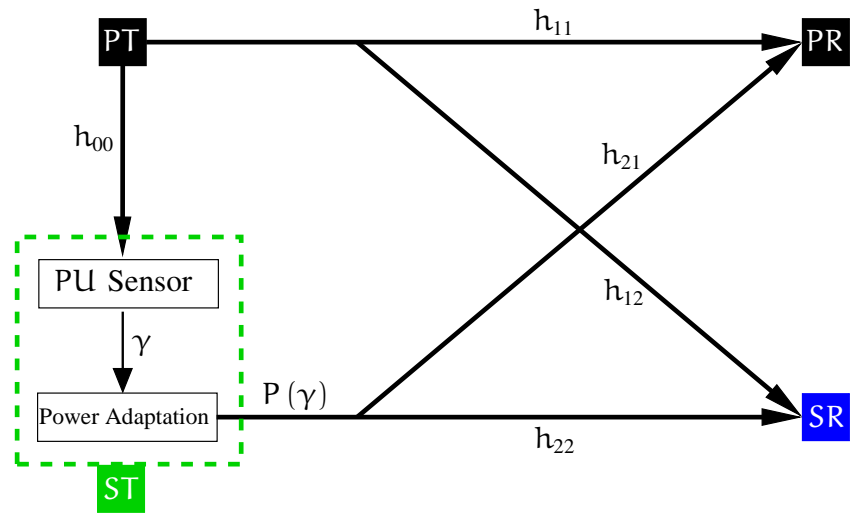

Fig. 1: System Model.

The channel coefficients between each of the primary and secondary nodes are considered to be independent Rayleigh distributed variables with variances that depend on the distances between the nodes, i.e.,

$$
h_{i j}=\operatorname{eN}\left(0, \frac{1}{d_{i j}^{2}}\right),
$$

where $d_{i j}$ is the corresponding distance between the associated pair of nodes. We assume no CSI at the transmitting nodes and perfect CSI at the receivers.

Every block, the primary user detector at the secondary transmitter monitors the frequency band for primary transmissions (Figure 1). Based on the signals received, the detector calculates a single sufficient sensing metric $\gamma$ as Figure 1 shows. To be as general as possible, we do not restrict the type of primary user detector, i.e., $\gamma$ can represent any sensing metric (for example, $\gamma$ can denote the total signal power observed, or the correlation between the observed signal and a known signal pattern, etc). We assume that the statistics of $\gamma$ conditioned on the primary user being ON/OFF are known $a$ priori at the secondary transmitter. We denote the distribution of $\gamma$ given that the primary user is OFF by $f_{0}(\gamma)$. Similarly, given that the primary user is $\mathrm{ON}, \gamma \sim \mathrm{f}_{1}(\gamma)$.

The secondary transmitter adapts its transmit power depending on the value of $\gamma$, i.e., if the value of the sensing metric in a certain block is $\gamma$, a power $\mathrm{P}(\gamma)$ is used to transmit the secondary signals for that block. We assume a peak power constraint at the secondary transmitter, i.e.,
Peak Power Constraint:

$$
\mathrm{P}(\gamma) \leqslant \mathrm{P}_{\max } \quad \forall \gamma .
$$

The secondary user is allowed to operate within the same frequency band as long as the average power received at the primary receiver (when the primary user is $\mathrm{ON}$ ) does not exceed a certain threshold $\mathbb{I}_{0}$, i.e.,

Average Interference Constraint:

$$
E_{\gamma} E_{h_{21}}\left[P(\gamma)\left|h_{21}\right|^{2} \mid P U ~ O N\right]=E_{f_{1}}[P(\gamma)] \frac{1}{d_{21}^{2}} \leqslant \mathbb{I}_{0},
$$

where $E_{f_{1}}[\cdot]$ denotes an expectation over the distribution $f_{1}(\gamma)$.

\section{A. Problem Statement}

The performance metrics of interest to us are the average SNR at the secondary receiver and the ergodic capacity of the secondary user. For the system model presented above, we seek answers to the following:

- Does soft sensing help improve the secondary user's SNR (or capacity)?

- What is the optimal power control strategy $\mathrm{P}^{*}(\gamma)$ that maximizes the secondary user's average SNR (or capacity)?

\section{Optimal Power AdAPTATION}

In this section, we consider the problem of secondary radio SNR and capacity optimization under the peak power (equation (2)) and average interference (equation (3)) constraints. Extensions to average power constraints and additional considerations such as channel knowledge availability at the secondary transmitter are explored in [10].

\section{A. SNR Maximization}

The average SNR at the secondary receiver $\xi_{s}$ can be written as in equation (4). I is a binary random variable that denotes whether the PU is $\mathrm{ON}(\mathrm{I}=1$, Prob $[\mathrm{I}=1]=\alpha)$ or OFF $(I=0$, Prob $[I=0]=1-\alpha=\bar{\alpha})$. Further simplification of equation (4) follows from the fact that $E_{h_{12}}\left[\frac{1}{1+P_{t}\left|h_{12}\right|^{2}}\right]=$ $\frac{d_{12}^{2}}{P_{t}} e^{\frac{d_{12}^{2}}{P_{t}}} \Gamma\left(0, \frac{d_{12}^{2}}{P_{t}}\right)$, where $\Gamma(\cdot, \cdot)$ is the incomplete Gamma function. Collecting the constants in $a_{0}=\frac{\bar{\alpha}}{d_{22}^{2}}$ and $a_{1}=$ $\left(\frac{\alpha d_{12}^{2} \Gamma\left(0, \frac{d_{12}^{2}}{P_{t}}\right)}{\bar{\alpha} P_{t}} e^{\frac{d_{12}^{2}}{P_{t}}}\right)$, the average SNR can be expressed as in equation (5).

The SNR maximization problem can be written as

$$
\max _{E_{f_{1}}[P(\gamma)] \leqslant I_{0}^{\prime}, 0 \leqslant P(\gamma) \leqslant P_{\max }} E_{f_{0}}[P(\gamma)]+a_{1} E_{f_{1}}[P(\gamma)],
$$

where $\mathbb{I}_{0}^{\prime}=\mathbb{I}_{0} d_{21}^{2}$. For the optimization of equation (6), we identify the power adaptation strategy $\mathrm{P}(\gamma)$ that maximizes the average SNR in the following theorem:

Theorem 1 (SNR Optimal Power Control): For a secondary user operating under the peak transmit power (equation (2)) and average interference (equation (3)) constraints, the power adaptation strategy that maximizes the secondary user's average $S N R$ is binary valued, i.e.,

$$
P^{*}(\gamma)=\left\{\begin{array}{ll}
P_{\max } & \text { if } f_{0}(\gamma) \geqslant\left(\lambda_{1}-a_{1}\right) f_{1}(\gamma) \\
0 & \text { if } f_{0}(\gamma)<\left(\lambda_{1}-a_{1}\right) f_{1}(\gamma)
\end{array},\right.
$$




$$
\begin{aligned}
\xi_{s} & =E_{h_{12}, h_{22}, \gamma, l}\left[\frac{P(\gamma)\left|h_{22}\right|^{2}}{1+\left.\left|P_{t}\right| h_{12}\right|^{2}}\right]=E_{h_{22}}\left[\left|h_{22}\right|^{2}\right]\left(\operatorname{Prob}[I=0] E_{\gamma \mid l=0}[P(\gamma)]+\operatorname{Prob}[I=1] E_{\gamma \mid l=1} E_{h_{12}}\left[\frac{P(\gamma)}{1+P_{t}\left|h_{12}\right|^{2}}\right]\right) \\
& =E_{h_{22}}\left[\left|h_{22}\right|^{2}\right]\left(\bar{\alpha} E_{f_{0}}[P(\gamma)]+\alpha E_{f_{1}}\left[P(\gamma) E_{h_{12}}\left[\frac{1}{1+P_{t}\left|h_{12}\right|^{2}}\right]\right]\right)=\frac{\bar{\alpha}}{d_{22}^{2}}\left[E_{f_{0}}[P(\gamma)]+\left(\frac{\alpha d_{12}^{2} \Gamma\left(0, \frac{d_{12}^{2}}{P_{t}}\right)}{\bar{\alpha} P_{t}} e^{\frac{d_{12}^{2}}{P_{t}}}\right) E_{f_{1}}[P(\gamma)]\right] \\
& =a_{0}\left(E_{f_{0}}[P(\gamma)]+a_{1} E_{f_{1}}[P(\gamma)]\right)
\end{aligned}
$$

where $\gamma$ is the soft information available from sensing and $\lambda_{1}$ is chosen such that equation (7) satisfies the average interference constraint (equation (3)).

Proof: See Section V-A.

Theorem 1 shows that a binary power control scheme is optimal, i.e., the secondary transmitter simply transmits at either of the boundary points ( 0 or the peak power $\left.P_{\max }\right)$ based on the roots of the equation $f_{0}(\gamma)-\left(\lambda_{1}-a_{1}\right) f_{1}(\gamma)=0$. Transmission does not take place at any intermediate power values. This result is somewhat surprising since it establishes that there is no SNR advantage to the soft information available from primary user sensing regardless of the sensing scheme or the form of the a priori probabilities. The soft sensing metric output from the sensing block can be replaced with a binary output without any loss in the average SNR while maintaining the interference level at the primary receiver.

\section{B. Capacity Maximization}

The ergodic capacity of the secondary user can be written as in equation (8) by conditioning on the value of I. The capacity optimization problem is: $\operatorname{Eax}_{\mathrm{f}_{1}[\mathrm{P}(\gamma)] \leqslant \mathbb{I}_{0}^{\prime}, 0 \leqslant \mathrm{P}(\gamma) \leqslant \mathrm{P}_{\max }} C_{s}$. The power adaptation scheme that maximizes the capacity is characterized in the following theorem:

Theorem 2 (Capacity Optimal Power Control): For a secondary user operating under the peak transmit power (equation (2)) and average interference (equation (3)) constraints, the power adaptation strategy that maximizes the ergodic capacity of the secondary receiver is given by equation (9), where $\gamma$ is the sensing metric. $\lambda_{1}$ is chosen to satisfy the average interference constraint.

Proof: See Section V-B.

Notice that unlike the SNR optimal power adaptation policy, the power adaptation that maximizes the capacity is, in general, not a binary one, i.e., it can involve transmission at non-boundary power levels between 0 and $P_{\max }$.

\section{Power Based Sensing}

In this section, we consider a power based sensing scheme and characterize the SNR maximizing power control strategy. The sensing metric is the total primary signal power in a number of independent signal samples, i.e.,

$$
\gamma(N)=\sum_{n=0}^{N-1}|y(n)|^{2},
$$

where $\mathrm{N}$ is the observation time. We assume that $\mathrm{N}$ is small compared to the primary user coherence time $T_{c}$. We consider the case of fast fading, i.e., where the channel coefficients change every sample. The received signal at the detector $y(n)$ is of the form

$$
y(n)= \begin{cases}h_{00}(n) x_{p}(n)+z(n) & \text { PU is ON } \\ z(n) & \text { PU is OFF }\end{cases}
$$

where $x_{p}(n)$ is the primary signal, $h_{00}(n)$ the coefficient of the channel between the primary and secondary transmitters, $z(n)$ the unit variance white Gaussian noise at the primary detector and $n$ is the sample index.

Notice that conditioned on the presence/absence of the primary user, $\gamma(\mathrm{N})$ is a sequence of independent and identically distributed random variables. When a primary signal is present, the sensing metric of equation (10) can be approximated by a Gaussian random variable (Central Limit Theorem) for large $\mathrm{N}$ with a distribution

$$
f_{1}(\gamma)=\frac{1}{\sigma_{1} \sqrt{2 \pi}} \exp \left(-\frac{\left(\gamma-\mu_{1}\right)^{2}}{2 \sigma_{1}^{2}}\right)
$$

where $\mu_{1}$ and $\sigma_{1}$ are given by

$$
\begin{aligned}
\mu_{1} & =\mathrm{NE}\left[|\mathrm{y}(0)|^{2}\right]=\mathrm{N}\left(\frac{\mathrm{P}_{\mathrm{t}}}{\mathrm{d}_{00}^{2}}+1\right) \\
\sigma_{1}^{2} & =\mathrm{N}\left(\mathrm{E}\left[|\mathrm{y}(0)|^{4}\right]-\left(\mathrm{E}\left[|\mathrm{y}(0)|^{2}\right]\right)^{2}\right) \\
& =2 \mathrm{~N}\left(\frac{\mathrm{P}_{\mathrm{t}}}{\mathrm{d}_{00}^{2}}+1\right)^{2}
\end{aligned}
$$

Similarly when there is no primary signal, the distribution $f_{0}(\gamma)$ can be written as

$$
f_{0}(\gamma)=\frac{1}{\sigma_{0} \sqrt{2 \pi}} \exp \left(-\frac{\left(\gamma-\mu_{0}\right)^{2}}{2 \sigma_{0}^{2}}\right),
$$

where $\mu_{0}=\mathrm{N}$ and $\sigma_{0}^{2}=2 \mathrm{~N}$.

Substituting (12) and (15) in equation (25), we have

$$
\frac{\left(\gamma-\mu_{0}\right)^{2}}{2 \sigma_{0}^{2}}-\frac{\left(\gamma-\mu_{1}\right)^{2}}{2 \sigma_{1}^{2}}+\ln \left(\frac{\sigma_{0}\left(\lambda_{1}-a_{1}\right)}{\sigma_{1}}\right) \leqslant 0,
$$

where the equality is satisfied with roots $\rho_{1}\left(\lambda_{1}\right)$ and $\rho_{2}\left(\lambda_{1}\right)$. Based on the discussion in Section III-A, the power adaptation can be calculated as follows:

$$
\mathrm{P}(\gamma)=\left\{\begin{array}{cc}
\mathrm{P}_{\max }^{*} & \gamma \in\left[\rho_{1}\left(\lambda_{1}\right), \rho_{2}\left(\lambda_{1}\right)\right] \\
0 & \text { elsewhere }
\end{array} .\right.
$$

The value of $\lambda_{1}$ is calculated based on the interference constraint at the primary receiver (equation (3)), i.e.,

$$
\begin{aligned}
\mathbb{I}_{0} d_{21}^{2} & =P_{\max }\left(\int_{\rho_{1}\left(\lambda_{1}\right)}^{\rho_{2}\left(\lambda_{1}\right)} f_{1}(\gamma) d \gamma\right) \\
& =P_{\max }\left(Q\left(\frac{\rho_{1}\left(\lambda_{1}\right)-\mu_{1}}{\sigma_{1}}\right)-Q\left(\frac{\rho_{2}\left(\lambda_{1}\right)-\mu_{1}}{\sigma_{1}}\right)\right)
\end{aligned}
$$




$$
C_{s}=E_{h_{12}, h_{22}, \gamma, l} \log \left[1+\frac{P(\gamma)\left|h_{22}\right|^{2}}{1+\left.\left|P_{t}\right| h_{12}\right|^{2}}\right]=E_{h_{22}, \gamma \mid l=0} \log \left[1+P(\gamma)\left|h_{22}\right|^{2}\right] \bar{\alpha}+E_{h_{12}, h_{22}, \gamma \mid l=1} \log \left[1+\frac{P(\gamma)\left|h_{22}\right|^{2}}{1+P_{t}\left|h_{12}\right|^{2}}\right] \alpha
$$

$P^{*}(\gamma)= \begin{cases}0 & \text { if } \bar{\alpha} f_{0}(\gamma) \frac{1}{d_{22}^{2}}+\alpha f_{1}(\gamma) \frac{1}{d_{22}^{2}} E_{h_{12}, h_{22}}\left[\frac{1}{1+P_{t}\left|h_{12}\right|^{2}}\right]-\lambda_{1} f_{1}(\gamma) \leqslant 0 \\ P_{\max } & \text { if } E_{h_{22}}\left[\bar{\alpha} \frac{\alpha f_{0}(\gamma)\left|h_{22}\right|^{2}}{1+P_{\max }\left|h_{22}\right|^{2}}\right]+E_{h_{12}, h_{22}}\left[\alpha \frac{f_{1}(\gamma)\left|h_{22}\right|^{2}}{1+P_{t}\left|h_{12}\right|^{2}+P_{\max }\left|h_{22}\right|^{2}}\right]-\lambda_{1} f_{1}(\gamma) \geqslant 0 \\ P(\gamma) & \text { elsewhere. } P(\gamma) \text { is the solution to } \quad E_{h_{22} 2}\left[\bar{\alpha} \frac{f_{0}(\gamma)\left|h_{22}\right|^{2}}{1+P(\gamma)\left|h_{22}\right|^{2}}\right]+E_{h_{12}, h_{22}}\left[\alpha \frac{f_{1}(\gamma)\left|h_{22}\right|^{2}}{1+P_{t}\left|h_{12}\right|^{2}+P(\gamma)\left|h_{22}\right|^{2}}\right]-\lambda_{1} f_{1}(\gamma)=0\end{cases}$

The resulting SNR at the secondary receiver can be written as

$$
\begin{aligned}
\xi_{s}= & a_{0} P_{\max }\left(a_{1} \mathbb{I}_{0} d_{21}^{2}+\right. \\
& \left.\left(Q\left(\frac{\rho_{1}\left(\lambda_{1}\right)-\mu_{0}}{\sigma_{0}}\right)-Q\left(\frac{\rho_{2}\left(\lambda_{1}\right)-\mu_{0}}{\sigma_{0}}\right)\right)\right)
\end{aligned}
$$

It is difficult to analytically determine the capacity optimal power adaptation from (equation (9)). We instead provide some numerical results comparing the optimal power adaptation strategies for SNR and capacity.

\section{A. Numerical Results}

We consider the primary and secondary nodes to be located such that $d_{00}=4, d_{11}=3, d_{12}=\sqrt{17}, d_{21}=1$ and $d_{22}=1$. For the primary user, we assume that the average $\mathrm{ON}$ time is $\alpha=0.5$ and the transmit power $P_{t}=1$. The power based sensing scheme at the secondary user calculates the total power in $\mathrm{N}=20$ samples of the primary signal. We assume a peak power constraint of $P_{\max }=1$ at the secondary transmitter.

We first consider a case where the tolerable interference at the primary user is $I_{0}=0.075$ ( $15 \%$ of $\left.\frac{\bar{\alpha} P_{\max }}{d_{21}^{2}}\right)$ and plot the SNR optimal power adaptation in Figure 2. Notice that the thresholds $\gamma_{1}$ and $\gamma_{2}$ are both positive and give rise to the pulse shaped power adaptation function shown in Figure 2.

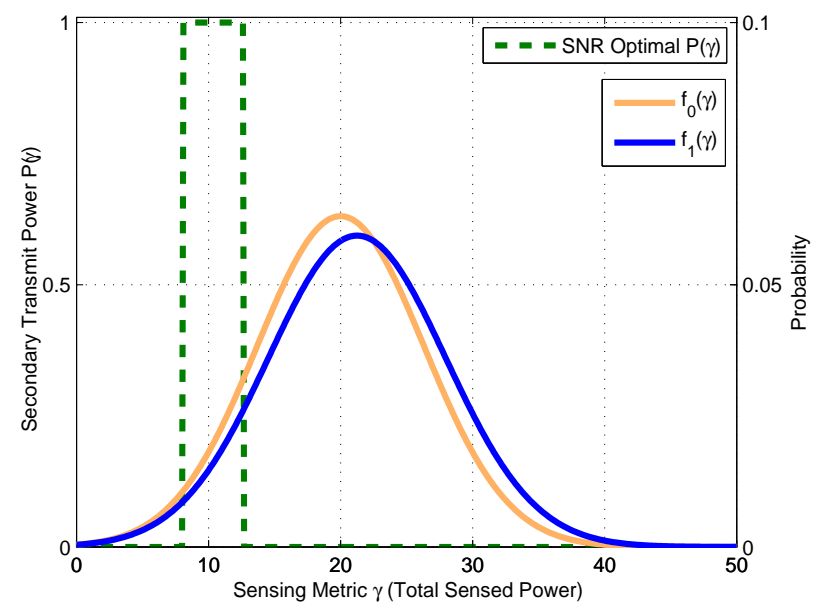

Fig. 2: Figure 2 shows the SNR optimal power adaptation with $15 \%$ (w.r.t $\frac{\mathrm{P}_{\max }}{\mathrm{d}_{22}^{2}}$ ) interference tolerance at the primary receiver.

We next consider a case where the $\mathrm{I}_{0}=0.05(10 \%$ of $\left.\frac{\mathrm{P}_{\max }}{\mathrm{d}_{21}^{2}}\right)$ and plot the SNR optimal and capacity optimal power

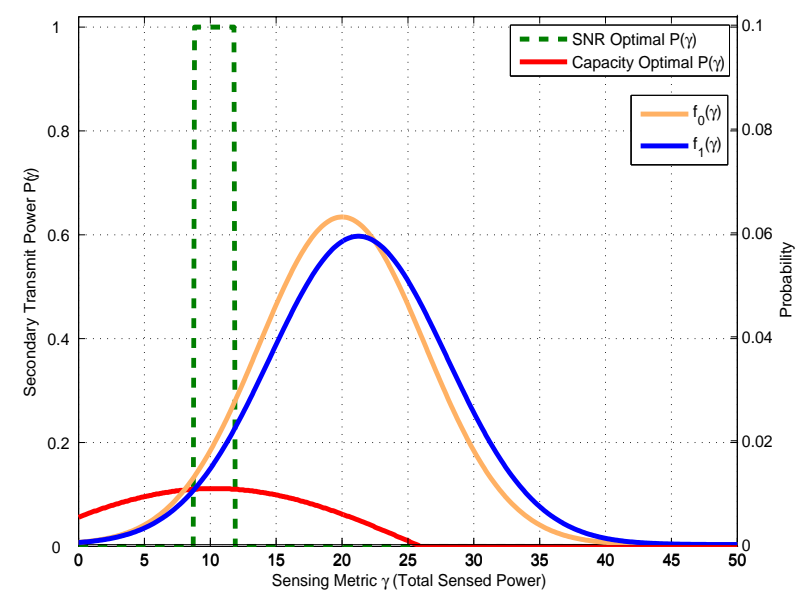

Fig. 3: Figure 3 compares the SNR and capacity optimal power adaptation with $10 \%$ (w.r.t $\frac{P_{\max }}{d_{22}^{2}}$ ) interference tolerance at the primary receiver.

adaptation policies in Figure 3. Notice that the SNR and capacity optimal power adaptation policies are markedly different. While the SNR optimal power adaptation policy is a binary strategy, i.e. mandates transmission either at zero power or at the peak power $P_{\max }$, the capacity optimal strategy involves transmission at intermediate power values. We explore the SNR and capacity dependence on the observation time $\mathrm{N}$ in [10].

\section{Proofs}

\section{A. Proof of Theorem 1}

The Lagrangian $L_{S}\left[P(\gamma), \lambda_{1},\left\{\lambda_{2}(\gamma)\right\},\left\{\lambda_{3}(\gamma)\right\}\right]$ for the objective function in the SNR maximization of equation (6) can be written as in equation (18), where $\lambda_{1}, \lambda_{2}(\gamma)$ and $\lambda_{2}(\gamma)$ are the Lagrangian variables.

It is easy to show that the objective function is concave in $\mathrm{P}(\gamma)$ and that the constraint set (equation (3)) is convex. Taking the derivative of $\mathrm{L}_{S}\left[\mathrm{P}(\gamma), \lambda_{1},\left\{\lambda_{2}(\gamma)\right\},\left\{\lambda_{3}(\gamma)\right\}\right]$ with respect to $P(\gamma)$ and setting it to zero, the necessary and sufficient KKT conditions are:

$$
\begin{gathered}
f_{0}(\gamma)+a_{1} f_{1}(\gamma)-\lambda_{1} f_{1}(\gamma)+\lambda_{2}(\gamma)-\lambda_{3}(\gamma)=0 \\
\lambda_{1}\left(E_{f_{1}}[P(\gamma)]-\mathbb{I}_{0}^{\prime}\right)=0 \\
\lambda_{2}(\gamma) P(\gamma)=0 \quad \forall \gamma \\
\lambda_{3}(\gamma)\left(P(\gamma)-P_{\max }\right)=0 \quad \forall \gamma
\end{gathered}
$$




$$
\begin{gathered}
\mathrm{L}_{S}\left[\mathrm{P}(\gamma), \lambda_{1},\left\{\lambda_{2}(\gamma)\right\},\left\{\lambda_{3}(\gamma)\right\}\right]=\left[\mathrm{E}_{\mathrm{f}_{0}}[\mathrm{P}(\gamma)]+\mathrm{a}_{1} \mathrm{E}_{\mathrm{f}_{1}}[\mathrm{P}(\gamma)]-\lambda_{1}\left(\mathrm{E}_{\mathrm{f}_{1}}[\mathrm{P}(\gamma)]-\mathbb{I}_{0}^{\prime}\right)+\int_{0}^{\infty} \lambda_{2}(\gamma) \mathrm{P}(\gamma) \mathrm{d} \gamma-\int_{0}^{\infty} \lambda_{3}(\gamma)\left(\mathrm{P}(\gamma)-\mathrm{P}_{\max }\right) \mathrm{d} \gamma\right] \\
\mathrm{L}_{\mathrm{C}}\left[\mathrm{P}(\gamma), \lambda_{1},\left\{\lambda_{2}(\gamma)\right\},\left\{\lambda_{3}(\gamma)\right\}\right]=\bar{\alpha} \mathrm{E}_{\mathrm{h}_{22}, \gamma \mid l=0} \log \left[1+\mathrm{P}(\gamma)\left|\mathrm{h}_{22}\right|^{2}\right]+\alpha \mathrm{E}_{\mathrm{h}_{12}, \mathrm{~h}_{22}, \gamma \mid l=1} \log \left[1+\frac{\mathrm{P}(\gamma)\left|\mathrm{h}_{22}\right|^{2}}{1+\mathrm{P}_{\mathrm{t}}\left|\mathrm{h}_{12}\right|^{2}}\right]- \\
\lambda_{1}\left(\mathrm{E}_{\mathrm{f}_{1}}[\mathrm{P}(\gamma)]-\mathbb{I}_{0}^{\prime}\right)+\int_{0}^{\infty} \lambda_{2}(\gamma) \mathrm{P}(\gamma) \mathrm{d} \gamma-\int_{0}^{\infty} \lambda_{3}(\gamma)\left(\mathrm{P}(\gamma)-\mathrm{P}_{\max }\right) \mathrm{d} \gamma \\
\mathrm{E}_{\mathrm{h}_{22}}\left[\bar{\alpha} \frac{\mathrm{f}_{0}(\gamma)\left|\mathrm{h}_{22}\right|^{2}}{1+\mathrm{P}(\gamma)\left|\mathrm{h}_{22}\right|^{2}}\right]+\mathrm{E}_{\mathrm{h}_{12}, \mathrm{~h}_{22}}\left[\alpha \frac{\mathrm{f}_{1}(\gamma)\left|\mathrm{h}_{22}\right|^{2}}{1+\mathrm{P}_{\mathrm{t}}\left|\mathrm{h}_{12}\right|^{2}+\mathrm{P}(\gamma)\left|\mathrm{h}_{22}\right|^{2}}\right]-\lambda_{1} \mathrm{f}_{1}(\gamma)+\lambda_{2}(\gamma)-\lambda_{3}(\gamma)=0
\end{gathered}
$$

For each value of $\gamma$, the optimal power adaptation $\mathrm{P}^{*}(\gamma)$ can be $0, P_{\max }$ or take a value in the open interval $\left(0, P_{\max }\right)$. This directly gives rise to the following three cases:

- Case 1: Suppose $\mathrm{P}^{*}(\gamma)=\mathrm{P}_{\max }$ for some $\gamma$, equation (23) requires that $\lambda_{2}(\gamma)=0$. Substituting this into equation (21) and noting that $\lambda_{3}(\gamma) \geqslant 0$, we have

$$
f_{0}(\gamma)+\left(a_{1}-\lambda_{1}\right) f_{1}(\gamma) \geqslant 0 .
$$

Therefore $P^{*}(\gamma)=P_{\max }$ for all $\gamma$ satisfying equation (25).

- Case 2: Suppose $\mathrm{P}^{*}(\gamma)=0$ for some $\gamma$, equation (24) requires that $\lambda_{3}(\gamma)=0$. Substituting this into equation (21), this is possible when (since $\lambda_{2}(\gamma) \geqslant 0$ ),

$$
f_{0}(\gamma)+\left(a_{1}-\lambda_{1}\right) f_{1}(\gamma) \leqslant 0 .
$$

- Case 3: Suppose $0<\mathrm{P}^{*}(\gamma)<\mathrm{P}_{\max }$ for some $\gamma$. From equations (23) and (24), we have $\lambda_{2}(\gamma)=\lambda_{3}(\gamma)=0$. From equation (21), we require

$$
f_{0}(\gamma)=\left(\lambda_{1}-a_{1}\right) f_{1}(\gamma)
$$

In general, the solution set to equation (27) (for a given value of $\lambda$ ) will have a measure of zero [10]. The power allocation at the roots of equation (27) will have to be expressed as impulse functions (i.e., of the form $\left.\mathrm{P}\left(\gamma_{0}\right) \delta\left(\gamma-\gamma_{0}\right)\right)$, that are excluded by definition because they do not satisfy the peak power constraint.

The optimal power allocation policy can therefore be written as in equation (7), where the value of $\lambda_{1}$ is calculated from the average interference constraint (equation (3)).

\section{B. Proof of Theorem 2}

The Lagrangian for maximizing the capacity function of equation (8) can be written as in equation (19). The derivative of the Lagrangian with respect to $P(\gamma)$ in equation (20) and the complementary slackness conditions of equations (22)-(24) form the KKT conditions for this optimization. As in the case of SNR, the three cases $\left(\mathrm{P}^{*}(\gamma)=\mathrm{P}_{\max }, \mathrm{P}^{*}(\gamma)=\mathrm{P}_{\max }\right.$ and $\left.\mathrm{P}^{*}(\gamma)=\mathrm{P}_{\max }\right)$ yield the three conditions of equation (9). A more detailed proof is provided in [10].

\section{CONCLUSION}

We consider a general cognitive radio system where the secondary transmitter adapts its transmit power depending on the soft information obtained from the spectrum sensor. We have a peak power constraint at the secondary transmitter and an average interference constraint at the primary receiver. We characterize the SNR and capacity optimal power adaptation strategies for arbitrary sensing schemes. Binary power control is SNR optimal, which shows that one can simultaneously obtain the dual benefits of optimum SNR performance and low power control complexity. On the other hand, the capacity optimal power adaptation scheme is, in general, not binary and dictates transmission at power levels other than 0 and $\mathrm{P}_{\max }$. For a power based spectrum sensing scheme, the SNR optimal power control scheme directs transmission at peak power if the sensing metric lies within a certain range.

\section{REFERENCES}

[1] National Telecommunications and Information Administration (NTIA), "FCC Frequency Allocation Chart," 2003. Download available at www.ntia.doc.gov/osmhome/allochrt.pdf.

[2] Aleksandar Jovičić and Pramod Viswanath, "Cognitive Radio: An Information-Theoretic Perspective," Submitted to the IEEE Transactions on Information Theory, April 2006. Available online at http://www.ifp.uiuc.edu/ pramodv/pubs/JV06.pdf.

[3] Natasha Devroye, Patrick Mitran and Vahid Tarokh, "Achievable Rates in Cognitive Radio Channels," IEEE Transactions on Information Theory, vol. 52, pp. 1813-1827, May 2006.

[4] Joseph Mitola, "Cognitive Radio: An Integrated Agent Architecture for Software Defined Radio," PhD Dissertation, KTH, Stockholm, Sweden, December 2000.

[5] Anant Sahai, Nigel Hoven, Shridhar Mubaraq Mishra and Rahul Tandra, "Fundamental Tradeoffs in Robust Spectrum Sensing for Opportunistic Frequency Reuse," Technical Report, March 2006. Available online at http://www.eecs.berkeley.edu/ sahai/Papers/CognitiveTechReport06.pdf.

[6] Sudhir Srinivasa and Syed Ali Jafar, "The Throughput Potential of Cognitive Radio: A Theoretical Perspective," IEEE Communications Magazine, May 2007.

[7] Syed Ali Jafar and Sudhir Srinivasa, "Capacity Limits of Cognitive Radio with Distributed and Dynamic Spectral Activity," IEEE Journal on Selected Areas in Communications, vol. 25, pp. 529-537, April 2007.

[8] Yunxia Chen, Qing Zhao and Ananthram Swami, "Joint Design and Separation Principle for Opportunistic Spectrum Access in the Presence of Sensing Errors," IEEE Transactions on Information Theory, February 2007. Submitted to the ITIT.

[9] Karama Hamdi, Wei Zhang and Khaled Ben Letaief, "Power Control in Cognitive Radio Systems Based on Spectrum Sensing Side Information," IEEE International Conference on Communications, June 2007.

[10] Sudhir Srinivasa and Syed Ali Jafar, "Soft Sensing and Optimal Power Control for Cognitive Radio," Journal paper in preparation, August 2007. 